\title{
The MINOS experiment
}

\author{
Gregory Pawloski ${ }^{* \dagger}$ \\ U Minnesota \\ E-mail: pawloskieumn.edu
}

MINOS/MINOS+ is an accelerator neutrino experiment that studies oscillation phenomena over a $735 \mathrm{~km}$ baseline by using the NuMI beamline. From 2005 to 2012, the MINOS experiment recorded beam data that was primarily taken in the low-energy NuMI configuration. After an extended shutdown the beam resumed operation in 2013 in a medium-energy configuration marking the start of operations for the MINOS+ experiment. In this paper, results using the MINOS and MINOS+ data samples are presented. These results include the measurement of three flavor oscillation parameters and limits on sterile neutrinos assuming a $3+1$ neutrino mixing model.

XIII International Conference on Heavy Quarks and Leptons

22-27 May, 2016

Blacksburg, Virginia, USA

\footnotetext{
*Speaker.

${ }^{\dagger}$ On behalf of the MINOS collaboration
} 


\section{Introduction}

The MINOS experiment was designed to measure neutrino oscillation phenomena governed by the atmospheric mass squared splitting by observing the transition of muon neutrinos and muon antineutrinos into other neutrino flavors over a baseline of $735 \mathrm{~km}$ along the NuMI beamline. These transitions can can detected by measuring a deficit of $v_{\mu}$ charged current (CC) and $\bar{v}_{\mu}$-CC interactions in the MINOS Far Detector, the appearance of $v_{e}$-CC and $\bar{v}_{e}$-CC interactions in the MINOS Far Detector, or the deficit of neutral current (NC) interactions in the MINOS Far Detector. From 2005 to 2012, the MINOS experiment recorded data in wide-band low-energy neutrino beam configuration with a peak at $3 \mathrm{GeV}$. In this beam configuration, the MINOS experiment is sensitive to the 3-flavor oscillation parameters: $\theta_{23}, \theta_{13}, \delta_{C P}$, and $\Delta m_{32}^{2}$. Furthermore, MINOS is sensitive to potential exotic phenomena such as nonstandard neutrino interactions and the mixing to sterile neutrino states. Starting in 2013, the neutrino beam began running in a medium-energy configuration that is peaked at $7 \mathrm{GeV}$ marking the start of the MINOS+ collaboration. In this higher energy configuration, MINOS+ can measure the neutrino transition probability at energies just above the first oscillation extremum at $1.6 \mathrm{GeV}$. This energy regime allows the experiment to not only test the energy dependence of the three flavor neutrino probability away from the minimum but gives the experiment a greater sensitivity to potential exotic phenomena that can lead to deviations in the high energy transition probability.

\section{NuMI Beam}

The neutrino beam is created at Fermilab by colliding $120 \mathrm{GeV}$ protons from the Main Injector onto a graphite target in the NuMI facility [1]. The charged particles produced in these collisions are focused into a beam by two magnetic horns and directed down a $675 \mathrm{~m}$ long decay pipe where the decays of pions and kaons results in the production of a neutrino beam. The relative position of the horns and the target as well as the horn current and polarity can be adjusted to tune the energy spectrum of the resulting beam. The beam primarily ran in a low energy neutrino mode for a recorded exposure of $11 \times 10^{20}$ protons on target with a composition of $91.7 \% v_{\mu}, 7.0 \% \bar{v}_{\mu}$, and $1.3 \% v_{e}+\bar{v}_{e}$. The beam also ran in a low energy antineutrino mode with a beam composition $39.9 \% \bar{v}_{\mu}, 58.1 \% v_{\mu}$, and $2.0 \% v_{e}+\bar{v}_{e}$ for an recorded exposure of $3 \times 10^{20}$ protons on target. Since September 2013 until the summer of 2016, the beam had been running in the medium energy neutrino configuration with a peak energy around $7 \mathrm{GeV}$ and an accumulated exposure of about $11 \times 10^{20}$. The results in this paper use at most $5.8 \times 10^{20}$ of the MINOS+ data set with the remaining data set to be analyzed later. A comparison of the resulting neutrino energy spectra for the medium-energy and low-energy NuMI configurations is shown in Fig. 1.

\section{MINOS Detectors}

The MINOS experiment consists of two detectors that are on-axis with the NuMI beamline. The 1 kiloton Near Detector is located $1 \mathrm{~km}$ from the production target and is used to measure the beam composition before the neutrinos undergo standard three-flavor oscillation. The 5.4 kiloton Far Detector is located $735 \mathrm{~km}$ from the production target and is used to measure changes in the 


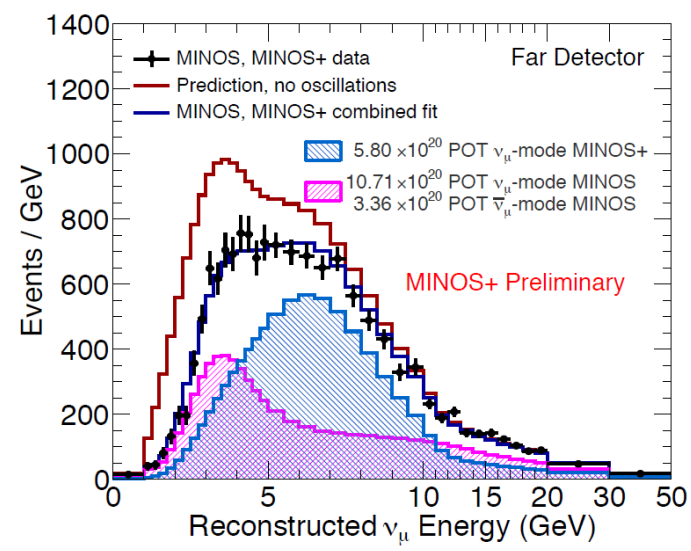

Figure 1: The reconstructed energy spectrum of $v_{\mu}$-CC candidate events in the Far Detector from the combined MINOS and MINOS+ data samples. The shaded magenta histogram is the best fitting MINOS contribution, and the shaded blue histogram is the best fitting MINOS+ contribution.

beam composition that are due to oscillation. In order to reduce uncertainties from flux, cross sections, and detector response, both detectors are functionally identical magnetized steel-scintillator tracking calorimeters [1]. The detectors have a planar design with each plane being composed of $2.54 \mathrm{~cm}$ thick steel layer and a $1 \mathrm{~cm}$ thick layer of plastic scintillator. The plastic scintillator is divided in $4.1 \mathrm{~cm}$ wide strips with the orientation of the strips rotated by $90^{\circ}$ for successive planes. Each strip has a wavelength shifting fiber embedded along its length and is read out by multi-anode photomultiplier tube.

\section{Results}

\subsection{Measurement of $\theta_{23}$ and $\Delta m_{32}^{2}$}

The MINOS experiment is capable of identifying $v_{\mu}$-CC interactions by measuring a reconstructed track that is consistent with a muon. The experiment can also identify $v_{e}$-CC interactions by using a multivariate analysis to determine if the scintillator pulse heights from a cluster of scintillator strips are consistent with energy deposited by an electromagnetic shower. By analyzing the energy distribution of $v_{\mu}$-CC and $v_{e}$-CC interactions in both beam data and atmospheric data, the experiment measures the values of $\theta_{23}$ and $\Delta m_{32}^{2}$ assuming three flavors. The resulting contours are shown in in Fig. 2. Most of the sensitivity comes from the observed disappearance of $v_{\mu}$-CC events in the beam. The ratio of the observed Far Detector beam data to the prediction with no oscillation is shown in Fig. 3.

\subsection{Search for Sterile Neutrinos}

The MINOS experiment can potentially observe the existence of sterile neutrinos by measuring neutrino oscillation that is governed by a fourth mass squared splitting, $\Delta m_{41}^{2}$. The most sensitive channel through which MINOS can observe this behavior is by measuring the disappearance of muon neutrinos into a sterile state by looking for a anomalous disappearance of $v_{\mu}$-CC and $v_{\mu}$-NC interactions which depends most strongly on the $3+1$ mixing matrix parameter, $\theta_{24}$. In order to 


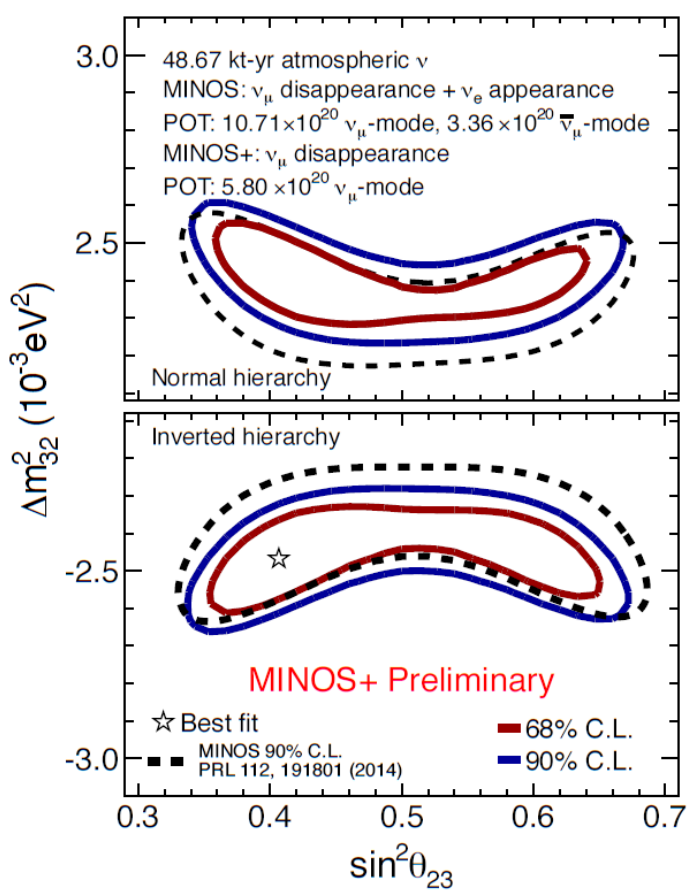

Figure 2: The allowed values of $\theta_{23}$ and $\Delta m_{32}^{2}$ at $68 \%$ and $90 \%$ CL using beam and atmospheric data from the combined MINOS and MINOS+ data samples. The MINOS contours are overlaid with the $90 \% \mathrm{CL}$ contours from the T2K [3] and NOvA [4] experiments.

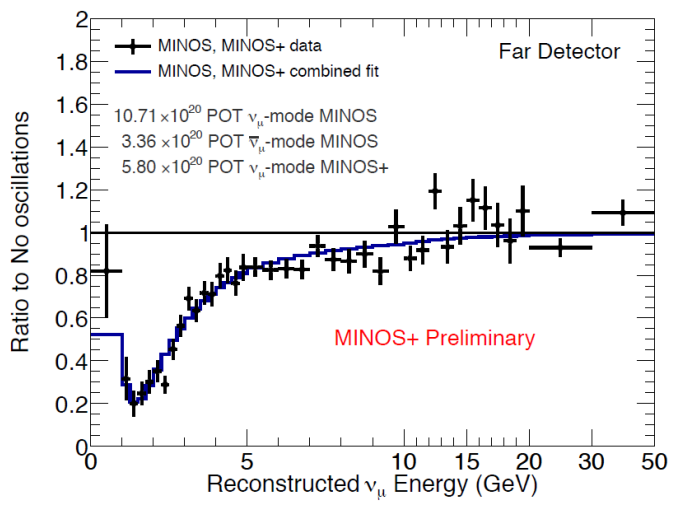

Figure 3: The ratio of selected $v_{\mu}$-CC events in the Far Detector over the prediction with no oscillation as a function of reconstructed energy from the combined MINOS and MINOS+ data samples.

take into account potential sterile mediated oscillation that may occur in both the Near Detector and the Far Detector, the ratio of the Far Detector selected events to the Near Detector selected events is measured and fitted assuming a $3+1$ neutrino model. The observed ratio for $v_{\mu}$-CC and $v_{\mu}$-NC interactions is shown in Fig. 4 using only MINOS data. These ratios are consistent with a three-flavor model and lead to limits being set on the possible values of $\theta_{24}$ which is shown in Fig. 5.

If one were to interpret the LSND [5] result in a 3+1 model, the LSND appearance signal 


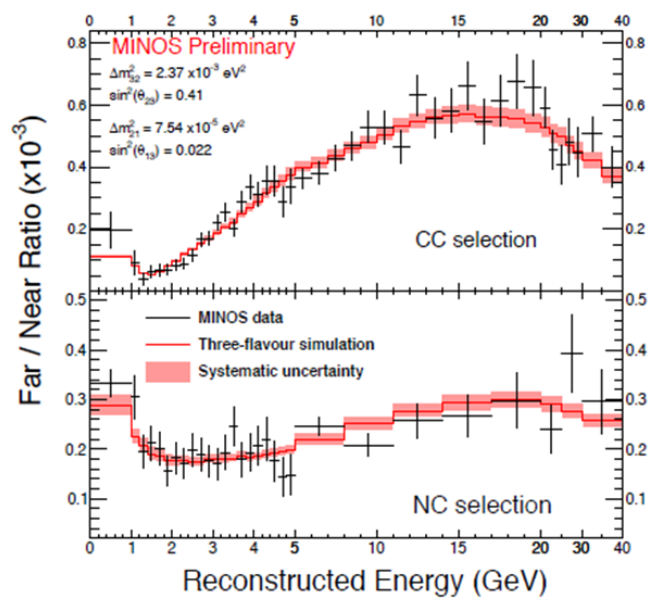

Figure 4: The ratio of selected $v_{\mu}$-CC and NC events in the Far Detector over the Near Detector as a function of reconstructed energy from the MINOS data sample.

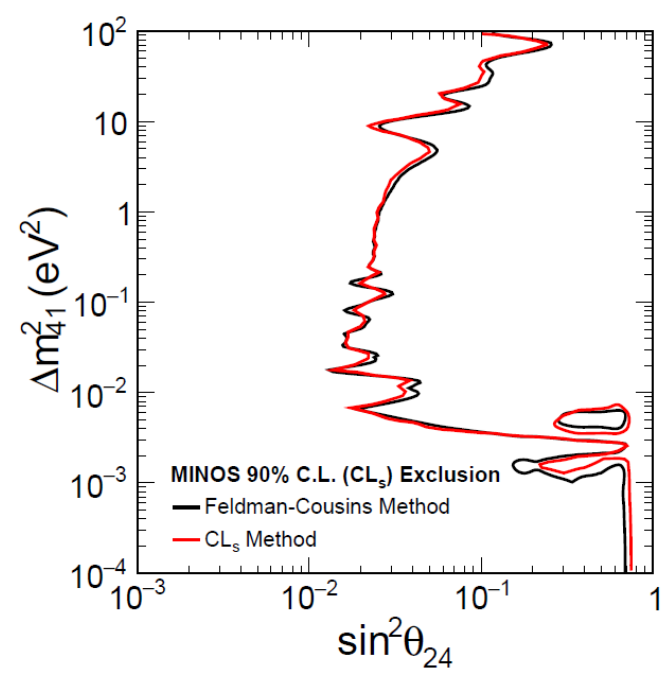

Figure 5: The allowed values of $\theta_{24}$ at $90 \%$ CL using the MINOS data sample.

would depend on the term $\sin ^{2}\left(\theta_{24}\right) \sin ^{2}\left(2 \theta_{14}\right)$. The constraints on $\theta_{24}$ from MINOS are then combined with the constraints on $\theta_{14}$ from the Daya Bay experiment [6] and Bugey-3 [7] to produce an exclusion of the parameter space allowed by LSND as shown in Fig. 6. The MINOS+ data set is currently being analyzed, but a preliminary plot of the ratio of the Far Detector selected events to the Near Detector selected events is shown in Fig. 7.

\section{Conclusion}

The MINOS experiment has produced contours of the allowed values of $\theta_{23}$ and $\Delta m_{32}^{2}$ that are competitive with the current results from T2K and NOvA. Using the MINOS low energy data, the MINOS experiment places the tightest constraints on $\theta_{24}$ at low values of $\Delta m_{41}^{2}$. By combining this 


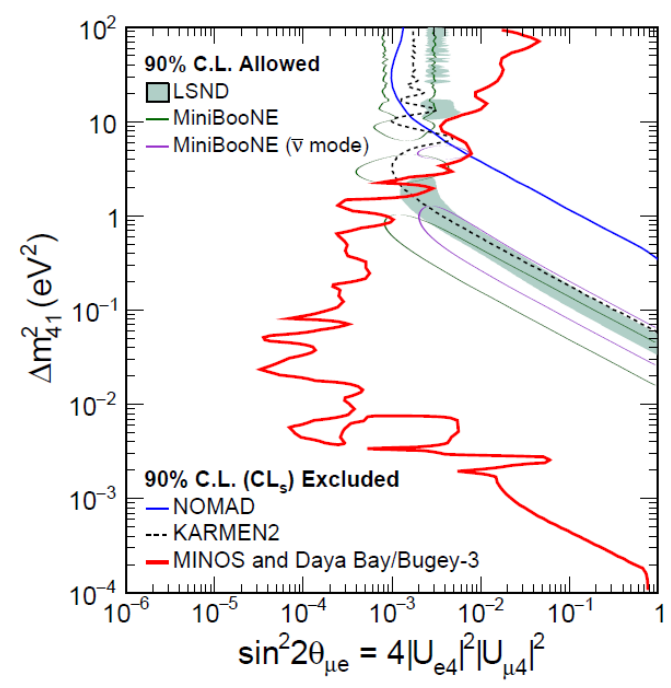

Figure 6: The exclusion of the LSND parameter space using the MINOS, Daya Bay, and Bugey-3 results. Overlaid with this plot are the allowed values from LSND and MiniBooNE [8] and limits from NOMAD [9] and KARMEN [10].

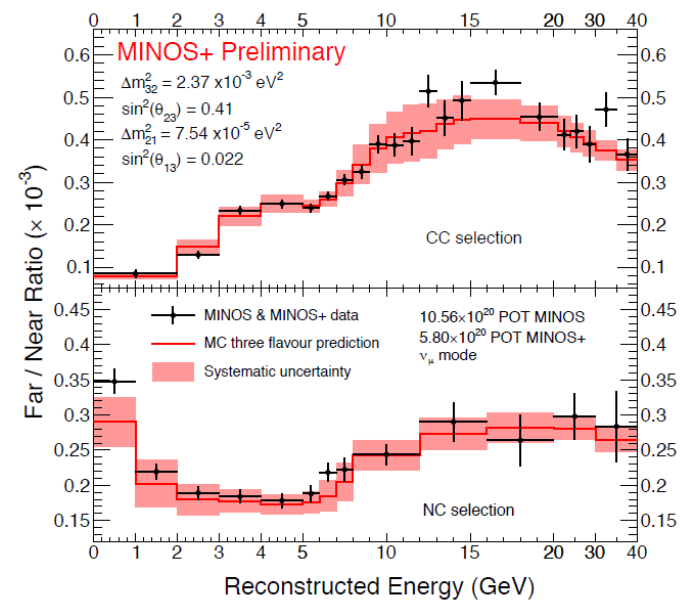

Figure 7: The ratio of selected $v_{\mu}$-CC and NC events in the Far Detector over the Near Detector as a function of reconstructed energy from the MINOS+ data sample.

result with Daya Bay and Bugey-3, the MINOS experiment excludes most of the allowed LSND parameter space. The MINOS+ data is currently being analyzed for sterile oscillation and will place even tighter constraints on the sterile neutrinos.

\section{References}

[1] P. Adamson et al., Nucl.Instrum.Meth. A 806, 279 (2016).

[2] D. G. Michael et al., Nucl.Instrum.Meth. A 596, 190 (2008).

[3] K. Abe et al., Phys.Rev.Lett. 112, 181801 (2014). 
[4] P. Vahle, Neutrino 2016 Conference, London (2016).

[5] A. Aguilar et al., Phys.Rev. D 64, 112007 (2001).

[6] F. P. An et al., Phys.Rev.Lett. 113, 141802 (2014).

[7] B. Achkar et al., Nucl.Phys. B 434, 503 (1995).

[8] A. Aguilar-Arevalo et al., Phys.Rev.Lett. 110, 161801 (2013).

[9] P. Astier et al., Phys.Lett. B 570, 19 (2003).

[10] B. Armbruster et al., Phys.Rev. D 65, 112001 (2002). 